\section{汉语跨文化语境之非线性 因素分析}

\section{Nonlinear Factors Analysis of Chinese Cross-Cultural Context}

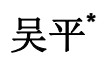

wuping0963@163.com

北京第二外国语学院汉语学院

北京 中国, 100024

Beijing International Studies

University

School of Chinese Language

Beijing, China 100024
汉语国际教育语境是一种跨文化的语境, 其特殊性体现出结构多样化, 层次多重性。这 种语境在时间、场合、对象、任务、心理、文 化等方面具有诸多的差异性和多变性, 这些因 素并非是有序排列和运行的, 具有独特性、开 放性和自由性等非线性特点。语言系统常因系 统内部一些细微的不确定性因素或来自系统之 外的某些微小因素而受到干扰，就可以导致大 量的、不可预测的波动。汉语自身和汉语教学 过程中, 存在着大量的非线性问题, 无法完全 用线性分析的方法来解决, 必须对汉语和教学 的非线性因素加以分析和研究, 以促进汉语教 学的发展。

[关键词］汉语国际教育；跨文化语境；非线性 因素分析; 浑沌学

* [作者简介] 吴平, 博士, 北京第二外国语大学汉语学院教授, 硕士生导师。研究方向: 对外汉语教学研究、汉语本体研究、文化语言学、修辞学。
The context of Chinese international education is a kind of cross-cultural context, and its particularity reflects the structure diversification and multiplicity. This context contains diversity and dynamics in the aspects of the time, place, object, task, psychology, culture and the like. These factors are not arranged and operated in an ordered way, but are endowed with the nonlinear characteristics of uniqueness, openness and polytrope. Language system is often interfered by the internal subtle uncertainty or some minor factors from external interference, can lead to a lot of, unpredictable fluctuations. In Chinese language and Chinese language teaching, there are a lot of nonlinear problems, which cannot be solved by linear analysis. Therefore, the analysis of non-linear factor in Chinese language study is very essential for promoting the development of Chinese language teaching.

Key words: Teaching Chinese to Speakers of Other Languages; cross-cultural context; nonlinear factors analysis; Chaos Theory. 
王希杰（1994）提出语言可以分为潜显两个层次, 第一个层次是显语言, 即人们平时所说的、所听到的、所看到的一切语言现象的总和。由于是在社会、 文化影响制约下逐步形成并不断演化, 具有非系统性和不对称性特征, 这其中, 几乎没有一条规则没有例外, 当然每一条例外中必隐含着另一条规则。第二个 层次是潜语言, 这是人们从未说过、听过或看过的, 但确实存在着, 随时可能 为人们所使用。正由于有潜语言的存在, 语言才可能具有开放性和生成性。潜 语言像一只蝴蝶一样, 在某年某月某一天的某个地方, 轻轻地煽动一下翅膀, 就会给语言带来极大的影响。语言教学, 尤其是作为第二语言教学/外语教学, 可以用以下相关的三个关键词开展讨论：汉语教学、跨文化语境和浑沌学。

\section{1. 浑沌学理论与二语学习}

“浑沌 (chaos)”一词中西皆有, 中国古代用来描述阴阳未分、天地合一、 万物伊始的宇宙状态。西方语言中的 “浑沌”一词源自希腊语, 原意是指先于 一切事物而存在的广衰虚无的空间, 后来被诠释为原始的混乱和不成形的物 质，宇宙的创造者就是用这种物质创造出了秩序井然的宇宙。但现代科学意义 上 “浑沌” 的概念并非表示 “混乱、无序”，而是建立在严格缜密的数学框架 之上的。浑沌理论认为: 所有的系统都遵从于不可预测的扰动, 浑沌系统不是 混乱的系统, 而是 “有序和无序、确定性和不确定性、稳定性和不稳定性的矛 盾统一, 因而不是简单的无序, 而是一种复杂的高级有序”（苗东升、刘华杰, 1994）。

浑沌学是 20 世纪初在自然科学中与相对论、量子力学几乎同时产生的新 学科, 科学家首先在自然科学领域发现存在着既确定又随机的浑沌现象及其非 线性规律, 并最终形成了浑沌/复杂性理论（Chaos/complexity Theory）, 这 是一门以直观、整体为基点来研究事物浑沌状态和浑沌运动的复杂规则性的学 问（张公瑾、丁石庆，2004：101）。浑沌学研究复杂的、非线性系统, 研究 无序如何变成有序、复杂现象如何在自然中产生, 并试图解释无序中的有序现 象, 探讨浑沌现象中各因素、各系统之间的相互影响。浑沌学理论特别强调浑 沌中的有序和有序中的浑沌之辩证关系。

语言学同人文社会科学、自然科学的外部横向交叉将促进语言学本身的发 展, 多学科的交叉已成为语言学发展的普遍趋向。浑沌学理论不仅在自然科学 研究中得到广泛应用, 它也推广到经济学、人类学、艺术学、社会学等人文社 会科学领域。为此, 张公瑾 (1997) 指出: “传统语言学的线性分析方法已经 不够用了, 我们需要有新的概念和新的方法来分析语言体系演变的复杂性, 对语言的演化及语言各种构件变化的随机性做出合理的解释。” 吴平（1998） 
认为, 混沌学之于语言学有非常广阔的应用前景, 而且可以适应于语言各个层 面的分析。浑沌学理论是第二语言学习和跨文化交际的需要。

Lightbown（1985）认为第二语言习得不是简单的呈线性累积发展，学习 者掌握了一些语法规则后, 又遇到新的语法规则, 相互冲突的语法规则导致整 个系统的重新构建。另外，长期生活在第二语言环境中的母语者也会产生母语 技能退化和丧失的现象。这些现象被称为 “语言耗损”（language attrition）。

Freeman (1997) ${ }^{1}$ 探讨了浑沌学理论应用于二语习得研究的前景。在详尽 讨论了二语习得的各种特性后, 她发现, 在决定一个习得者的中介语发展的过 程中，有许多相互作用的因素。在语言因素方面涉及源语（source language） /母语、目的语、母语的标记性、二语的标记性、输入语的数量和种类、交互 作用（interaction）的数量和种类、学习者接受的反馈数量和种类, 以及二语 习得的语境是课堂内还是课堂外等。在学习者个体因素方面包括年龄、语言潜 能（aptitude）、动机和态度等社会心理因素。此外, 性格因素、认知风格、 学习策略、性别、出生次序（birth order）、动机、兴趣等也都可能产生影响。 这些因素就是语言习得系统中的 “奇异吸引子 (strange attractor) ${ }^{2}$ ”, 它们的 交互作用使得本来微小的差别可能随着时间的推移不断放大, 然而这不是按固 定的线性方式变化，且最终会放大到显著的差距程度，造成学习者之间巨大的 差异。而二语习得中的僵化现象有可能是由于中介语变得封闭, 转向了一个固 定点的吸引子所致。她认为二语习得同样是一个复杂的、动态的、非线性的过 程。她认为, 这种对动态的、复杂的、非线性系统的研究对于二语习得研究也 有意义。Freeman 认为 “目的语” 这个词其实是误导的，因为学习者的习得 过程是没有止境的, 而且这个目标也是在不断移动变化的, 实际上它是一个非 线性的概念。一个学习者的中介语也是一个自我参照系统, 在某种程度上说, 它可以用规则来说明, 但并非由之产生的。它是一个经常变化并且会经常对它 接收到的回馈做出反应的系统。它也是一个开放系统, 而且会向奇怪吸引子移 动, 这个吸引子会给它一个动力。

在 2014 年召开的 TESOL 年会上, Freeman ${ }^{3}$ 所做的演讲详述了对语言 和语言教与学的新思考。基于对复杂理论的独到见解, 她提出了几个观点: 语 言系统是通过说话者之间、说话者与其所在环境之间不断的交流互动而形成

\footnotetext{
参见: 吴平, 2011 , 第二语言习得中的浑沌/复杂性理论 $[\mathrm{A}]$, 浑沌学与语言文化研究新 动态一一国外相关研究译文集 [C]。北京: 中央民族大学出版社, 149-160。

2 浑沌系统存在两个相反行为的吸引子, 即收玫性吸引子和奇异吸引子 (或称混沌吸引 子)。收玫性吸引子起到限制系统运动的作用，使系统的性态呈现出静态的平衡特征; 而奇异 吸引子则使系统偏离收玫性吸引子的区域而导向不同的性态，它通过诱发系统的活力，使其变 为非预设模式, 从而增加系统的创造性。奇异吸引子被视为影响系统运作的重要因素。
}

参见: 高洪德, 2015, 教语言, 还是教学习者 $[\mathrm{J}]$ 。英语学习・教师版, （5）：31-33。 
的; 语言系统的特点是变化，而不是恒定；语言发展有非线性特点，常 “因果 不对称”。在此基础上，她对二语学习中 “acquisition” 概念提出质疑，认为 “acquisition” 指单向行为, 而 “development” 则是互动过程。因此她认为 “第二语言发展”（SLD）能更为恰当地描述大家所研究的领域。同时, 她还 分析了 “input” 和 “affordance " 的关系, 指出前者是从环境到学习者的单 向行为, 语言成为固定语码, 学习成为记忆的过程。而后者可理解为 “可接受 性” , 是指学习者根据自身的理解方式感悟语言内容, 在易于他们理解的语境 中用他们可以承受的方式学习语言。Larsen-Freeman 提出的最后一个问题是: 教语言和教学习者的关系是怎样的? 她认为, 二语教学就是为学生创造多语言 环境（multilingual spaces）。在这环境里, 学生的社会文化身份得到认可, 学生原有的知识得到关注, 他们能成为积极的合作参与者, 能享受到如在家中 般的人格尊重。

Ellis（2004）指出二语习得与母语系统、中介语系统、学习者潜质、学习 者策略、教师指导、语言输入及互动活动等密切相关，是各种错综复杂的因素 交互作用的结果。

从浑沌学理论视角来看, 语言是一个多维开放的系统, 受社会、历史、文 化和政治等因素的影响; 语言教学亦非一个封闭静态的系统, 其内部的各个子 系统不断相互影响、相互作用, 并与外界各种社会文化因素一起促进教学系统 动态地发展; 语言和语言教学都具有复杂性、非线性、动态性和不可预测性等 特征。每个人都有自己的思维方式, 语言教学过程会因不同文化背景和不同学 习经历有所区别。学习者在学习过程中先受母语的影响, 再受第二语言及中介 语系统的影响。中介语系统的整体稳定而局部不稳定, 对初始条件（initial condition）有敏感的依赖性，初始条件的稍微改变，都会导致中介语发生不 可预测的变化。貌似有序的语言教学实际上是一个浑沌的非线性过程。在这种 浑沌的过程中产生了一种新的语言一一中介语。从浑沌学理论来分析, 母语是 二语习得的初始条件，母语和第二语言共同作为二语习得的奇异吸引子，它们 的结构相对稳定，对学习者的中介语有一种吸附的作用。因此，二语习得是一 个复杂的动态非线性系统。

\section{2. 从浑沌学角度看汉语}

语言是一个开放的、非线性的复杂系统, 用浑沌学理论来研究语言系统的 非线性特征及其浑沌行为是语言学发展的自然趋势。语言系统不是一个确定、

${ }^{4}$ 这是 James Gibson 提出的一个概念, 可译为 “可供性” , Gibson 认为这个概念跨越了 客观和主观的二分法, 既是物理的又是心理的, 也都不是; 它还包含了环境和观察者。这个概 念从二语教学角度来说，给我们提供了一种认识语言世界的方式，这比间接认知论（对刺激做 出反馈）更加接近人的真实认知。 
平衡、有序的模式, 它常因系统内部的一些细微不确定的因素或来自系统之外 的某些微小因素受到干扰，可以导致大量的、不可预测的波动。汉语自身特点 鲜明，存在着大量的非线性问题，无法完全用线性分析的方法来解决，必须对 汉语教学中的非线性因素加以认真分析和研究, 以促进汉语教学的发展。

拼音文字都是由字母从左至右或从右至左的线性排列组词。汉字不是, 汉 字是由不同部件组合而成的非线性结构体, 既可以是左右结构, 也可以是上下 结构; 还可以是左中右、上中下、包围、半包围、相交、相离等多种结构形态。

汉字既有独体字（如一、不、千），也有合体字（如：休、迁、包）；而 特殊的合体字更是蕴涵了中国文化特色，如：红双喜字（㰻），称之为 “吉利 字”、“吉语字” 和 “吉祥合体字” ，其源头可追溯至先秦时君主用来传达命 令或徵调军队的符文。通常是把几个管字合并在一起, 刻在竹子或木头上, 再 剖为两半, 双方各执一半, 合之以验真假。到了道教流行的汉代, 这种手法便 被道士们借用到所谓符管上，称为 “复文”。因为合体字长期被应用在道教符 銢上，而符籙一向被认为带有召鬼神、镇精魅的奇特力量; 民间亦深受其染， 造成以吉语合成文字的兴起。从宋代起, 合体字已从道士画符, 演化为老百姓 表达避凶求吉愿望的一种手段。如: 招财进宝、黄金万两、日日有财、福禄寿 全、好学孔孟、唯吾知足等, 并被广泛用于商铺、节日、婚嫁喜庆之时。再如: “biangbiang” 面是源于陕西西安的一种面, 现在也是一家面馆的品牌, 还在 使用这个字，这个字普通字典中查不到，但康熙字典里能查到。

陕西关中 “biangbiang” 面文字儿歌 (有许多的版本) 这样唱到:

“一点上了天，黄河两道湾，八字大张口，言字往进走，

你一扭我一扭, 你一长我一长, 当中加个马大王, 心字底月字旁,

挂个丁丁叫马杠, 坐着车车逛咸阳。”

汉语在构词造句时, 缺少形态变化, 仅有一些类语缀存在, 大多数汉字 (实 字) 可以自由组合, 或左或右复合成词。如有些由两个意义相近的字组成的词, 把它颠倒过来念, 意思基本保持不变: 情感——感情、蔬菜一一菜蔬、语言一 一言语等等; 有的词语两个语素颠倒后, 意义仍然相近或相关联: 蜜蜂一一蜂 蜜、彩色——色彩、奶牛一一牛奶等等; 还有不少由两个意思相近的字组成的 词语意义发生了改变, 如: 气节——节气、人名一一名人、人情——情人等等, 这类词语如果颠倒了它们的意思就完全不同或相差很远; 还有一种更有趣的现 象, 就是有些东西的名称, 如果把它倒过来说, 正好表明了这种东西的用途, 例如: 门锁——锁门、牙刷——刷牙、鱼网——网鱼等等。

5 参见: 吴平, 2008 , 同素反序双音节名词中的浑沌现象分析 [A], 混沌学与语言文化研究 新视野 $[\mathrm{C}]$ 。北京: 中央民族大学出版社。 
意合成句。“可以清心也” 算得上是一个汉语意合特征的典型例证：可以 清心也，也可以清心，心也可以清，清心也可以，以清心也可。

分形（fractals）是由于具有不规则的比例自相似性，如果在复杂的图形 中取出一部分放大到原型大小后, 看上去仍具有原图形的典型特征, 这说明可 以通过认识部分来映像整体。分形的复杂性来自简单数学关系的反复迭代, 源 于局部与整体的自相似性, 自相似性是一种普遍存在并把它作为一种由已知到 未知的思维模式, 可以由小到大也可以由大及小, 可以由表及里也可以由内到 外。汉民族的传统思维特点是一体思想。把自然、社会和人视为一个和谐的统 一体，因而被称为是经验综合性的整体思维、直观思维和辩证思维。对应到汉 语的特点就有其 “自相似性”。

汉语词汇发展上，给某些具有同一属性的事物以一个 “总名”。古代的江 河都有专名, 如江、河、渭、汉、湘、淮等, 并加 “水” 以表示河流，在许慎 的《说文》中，用 “水” 作为通名，使之成为众多河流的概括性称呼。

从词的理据上看，汉语给事物命名时，常对事物从直观上作整体把握，利 用人和事物的相似特征进行类比，并尽可能的给予相同的命名（只是在字形上 予以区别），如人的头顶叫 “颠”，山顶叫 “巅”，树顶叫 “槙”。

语言的发展对路径和规则的选择有依赖性,一旦选择了某条路径就很难改 变, 一旦形成规则就不易改弦易辙。汉语中对于空间顺序的选择是由大及小, 英语则是由小到大。汉语方位中有 “东南、东北、西南、西北” , 英语则是 “southeast、southwest、northeast、northwest”。汉语对时间顺序的选择 是由整体到个体，这里就既有汉民族认知方面的特点，也有文化上的传统。

当一个系统处于结构变革时期时, 其体系就属于结构耗散系统, 就会出现 一些按常规常理难以解释的奇怪现象，其中的 “奇异吸引子” 代表系统中的稳 定态, 它对周围有吸引作用, 系统运动只有到达吸引子的范围才能稳定下来并 保持下去。新词新语是词汇系统中的一个动态系统，“出租汽车” 本来也算是 一个新词语, 但随着 “的士” 的出现, “的” 的口语色彩越来越浓厚, 以“的” 为构词成分的新词也大量出现：打的、面的、摩的、的哥、的姐…… “的” 还 可以这样用: “他每天都是的来的去” ，意思是他每天都坐出租汽车来往。

\section{3. 二语教学（汉语国际教育）是一个复杂、浑沌的系统}

二语（汉语）教学中, 虽然我们有一个线性的教学大纲、教材以及限定的 课堂教学, 教师还针对特定教学对象制定了较为详尽的教学计划、教学进度和 教案, 但是学习语言的过程并非一个线性的过程, 因为学习者并非先掌握了一 个语言技能, 然后再转到另外一个语言技能。事实上, 即使是学习某一个单项 的语言技能，这个学习过程本身也是非线性的。在决定一个习得者的中介语发 展过程中, 有许多相互作用的因素。在语言学习者的系统里, 语言系统之间有 
一个互动作用。当一个习得者开始学习一种新形式时, 以前掌握的形式就会变 得不稳定。同样, 学生在新的情景里看到的规则会暂时改变他对这个新规则的 理解。我们也要注意到复杂系统的动态模型会表现出反馈圈, 这种反馈圈在生 物进化中已经被接受, 也可以用于学习。他们吸收外部影响, 并且改变内部结 构来适应新环境。在生物学上, 这种因素是单个的有机体, 回馈是自然选择提 供的, 这种模式的稳步改善被称为进化。但是在学习语言的认知过程中, 这种 过程实际上也是相同的, 有机体变成了单个的个体, 反馈则来自于老师和学生 自己的直接经验。复杂系统是自我参照系统, 而秩序产生在这些看似浑沌的体 系, 被 “奇异吸引子” 的力量所吸引。由于这些系统极端复杂, 秩序就会从最 简单的规则里产生, 正是这些规则把系统内的相互作用等同为一个整体; 而这 种个体的组成部分的相互作用也会产生出稳定性。

第二语言教学存在许多随机和不稳定因素, “课堂教学和其他生命体一样 都要经历浑沌的无序与有序”（Freeman, 1997：141）。然而在教学实践中, 语言学习常常被错误地看作一个语言知识和交际能力递增的线性过程, 教完 这个知识点之后又教下一个知识点, 认为学生能够逐个学会这些知识。事实上, 语言课堂教学是一个非线性的复杂系统, 课堂的诸种要素交互作用, 一些变化 是可以预测的, 而另一些变化又是不可预测的, 这就要求教师提高教学能力, 更好地驾驭课堂。在二语教学过程中, 同一个教师教学, 不同学生的学习效果 却相差很大; 同一个教师上课, 不同班级的学习表现也大不一样。其实, 课堂 是一个复杂多变、动态发展的开放系统。每一位学生的语言领悟能力、语言学 习态度和动机、语言学习策略和语言学习经历等都有较大的差异, 对来自课堂 上的知识和情感信息做出的反应不同, 大脑对信息加工的过程也不相同。语言 学习者之间的差异还会随时间的推移而不断扩大, 并以非线性的方式递增, 学 习效果也就自然会有差别。在这个复杂的教学系统中, 许多现象是处于非线性 状态, 无法预测, 但在杂乱无序的交互活动中又形成一些复杂的规律, 这些规 律让语言学习成为了可能。

二语学习的 “停滞不前” 现象。对于二语学习者来说, 其二语水平到了某 一阶段便停滞不前甚至呈现下降趋势, 这种不可预测、非线性的表现, 源于学 习者个人心理、生理、语言学习能力等个人特征、所学语言及其所处的学习环 境, 如教材、教法、教师、学习氛围等变量之间交互影响, 从而形成了错综复 杂的浑沌状态。浑沌学理论认为这是偏离秩序的浑沌状态, 浑沌学理论将二语 学习视为一个开放的复杂系统, 该系统与其他相关系统相互作用、相互影响, 并呈现复杂变动的现象和结果。需要特别指出的是, 学习者在学习过程的各种 变量经过重新组合, 交互作用, 重新恢复秩序, 进入新的有序状态。

二语 “石化” 的现象。学习者在中介语连续体尚未达到目的语状态时便停 止发展, 某些语言错误已作为一种语言习惯固定下来, 进一步的学习也无法改 变。不少汉语学习者在学习过程中反复出现的某种错误, 如汉字书写和认读、 语音、词语、语法、语用等, 若得不到及时的彻底的纠正, 最终成为 “化石”, 
之后往往再努力也无济于事, 严重影响到学习的效果和之后的学习, 这就是 二语学习过程 “蝴蝶效应” 的表现。二语习得中 “石化” 现象的发生是由于中 介语变得封闭, 转向了一个奇异吸引子的缘故所致。当然, 二语学习过程中的 各种变量经过交互作用, 从无序状态重新恢复秩序。

二语的 “学习效果”现象。二语学习始终离不开学习效果或者说学习成绩, 好的效果是几乎接近或者基本接近目的语, 这是一种理想的结局, 但二语学习 者学习过程的非线性还表现在学习者策略的运用与学习成绩之间的关系上, 即 付出了大量的劳动, 收到的是 “事倍功半” 的效果。学习策略的运用和学习策 略训练的有效性已得到证实。对英语学习者的研究表明学习策略与学习成绩之 间存在线性关系，例如: Green 和 Oxford 在他们的研究中发现两者之间存在 统计学上的正相关。 ${ }^{6}$ 由于学习过程涉及到复杂多变的因素, 如学习者面对不 同的任务、内容、学习者的学习水平和阶段、学习动机和心理、学习环境等等 都会对学习策略的运用造成影响, 使学习过程处于浑沌状态, 也就造成学习策 略的运用与学习成绩的非线性。非线性在策略的训练上表现为: 有的学习者经 过一段时间的训练后，尽管他们意识到学习策略的有效性，但在实际使用语言 过程中又回复到以前的旧方法。策略培训应该考虑到不可预测性和复杂性，根 据不同的情形加以调整变动，这是教师和学习者都必须重视的问题。

当代主流教学理论认为, 教学活动是由教师、学生、教材三大基本要素构 成的, 它们组成了一个相对稳定的教学活动结构, 并成为判断教学活动区别于 其他社会活动的基本依据。但事实上，教学活动系统是由这三大基本要素构成 的吗？教学活动的复杂性主要表现在人 (教师与学生) 的复杂性与知识的生成 机制、知识状态与层次的复杂性。教师、学生、教材 (知识) 三者并不存在一 种稳定结构, 教学系统的要素应该是开放性的、生成性的、联性的、偶发性的。 或者说，教学活动只有在三者甚至更多复杂的关系中才能开展。

从某种程度上说, 教学现象与一般现象一样, 具有一定的客观性、确定性、 普遍性和可量化性, 可以通过观察、实验等方法对其进行实证和研究。但教学 更是一个复杂的、浑沌的系统。教学活动尤其是教师与学生的思维、情感、智 力和人格心理及行为不仅具有自然属性, 更具有的复杂的特点, 比如主观性、 偶然性、自我调适性、非量化性等。教学行为更多地是意义与价值的世界, 不 可能完全客观地加以描述, 都必须通过体验与理解等复杂的方法才能把握。

教学系统是一个线性和非线性统一的系统。因此, 在教学中, 我们既要看 到教学的线性特征也要看到教学中的非线性特征。从总体上来看, 教学必然带 来学生的发展, 教学是因, 学生的发展是果, 呈线性因果关系。但从局部来看, 教学和学生的发展表现为互为因果关系, 而且它们之间结合成一个密切联系而

6 转引自: 丁梦阳, 2008 , 非线性科学对外语学习及教学的启示 $[J]$ 。江苏教育学院学报, (1) $: 100$ 。 
不可分割的整体。在课堂教学中这种非线性特征表现得非常明显, 学生的发展 水平和其身心状况制约着教学, 而教学又调节和促进着学生的发展与身心状 况。课堂中的任一偶发事件或对教学目标的微小偏离, 都会给课堂教学系统带 来巨大的变化, 也会给学习的结果带来巨大的变化。

教学要坚持以学生为主体, 自主学习的原则, 在此前提之下, 应该关注学 习者的情感因素。这里的 “情感” 包含了学习者个体对于动机、兴趣、自尊、 自信、自我评价、自我形象、师生关系、生生关系、教学环境等所产生的态度 体验。它们扮演浑沌学中 “奇异吸引子” 的角色, 这些吸引子若得到充分的正 面发挥, 将极大地改善二语学习的进程, 提升学习效率和效果。二语学习活动 的每个环节、每种结果对此都带有敏感的初始依赖性: 情感作用发挥得好, 学 习者的学习积极性得到调动, 学习能力得到发挥, 潜力得到挖掘, 学习过程中 的心理、思想问题也随之得到解决。就汉语学习过程而言, 低龄或低年级学习 者, 由于种种原因, 对汉语一般较易产生畏难情绪, 若得不到及时的排遣、化 解和疏导, 或许他就此放弃努力。相反, 初学者的兴趣若能得到正确引导, 教 师若能为之营造积极的愉快的学习氛围, 他们的语言潜力或许就此得到较充分 的挖掘和发挥, 动机也由此得以激发, 整个教学活动就能处于良好的状态之下, 情感因素具有 “蝴蝶效应”。

因此, 如何激发和尊重学生的情感因素是汉语教师必须面对的重要课题。 这为提高汉语教师对汉语学习过程的理解提供了一个新视角。第二语言习得是 在熟悉和掌握了母语前提 (基础、条件) 下进行的, 它的语言学习背景与初学 母语时差别很大, 原有语言习得机制 (初始条件)对第二语言产生怎样的敏感性 作用, 教师应当具有一种自觉的探索精神和意在理解的态度。教师应该着眼于 发现和分析在许多表面学习现象和学习者行为背后隐含着的因素、细微的差别 变化以及学习过程中的其它初始条件, 观察并求解发生在教学临场不可预测的 事态及其千变万化的 “规则”, 以便充分地作好教学准备, 化教学中的 “无序” 为教学中的 “有序”, 通过反馈控制切实提高汉语教学效果与质量。

教学理论研究不存在像自然科学那样强烈的、普遍有效的、线性的、逻辑 化的因果关系, 必须借助于人与人的交往与语言来加以理解、解释与体现。在 教学活动中, 只有主观认识与作为 “文本” 的客观事物之间达成一致与融合, 才能获得教学的理解与解释。

“奇异吸引子” 的作用在于使系统偏离收敛性吸引子的区域而导向不同的 性态, 它通过诱发系统的活力, 使其变为非预设模式, 从而增强系统的创造性。 在课堂教学中, 学生各自独特的个性和独特的发展可能性, 便是奇异吸引子。 要使学生真正获得自身可能的发展, 就需要改变教学目标的一维性, 设置多维 的、多层次的教学目标, 这势必导致系统在总体上遵循教育目标的情况下, 在 局部范围内产生对教学目标的合理偏离, 也正是这一状态触发课堂教学系统局 部呈现出丰富多彩, 从而增强课堂教学的创造性。 


\section{4. 跨文化语境下汉语教师的资质与浑沌学}

周昌乐、丁晓君 (2000) 的研究发现, 语言理解过程中机器所面临的主要 困难, 包括意群分割, 歧义消除, 语言、文化和心理多层次解读以及对言外意 义的领悟等。对以机器理解汉语的困难分析来看, 一个突出的关键问题就是, 所有这些困难在人类语言理解中的实现, 都离不开整体语境的作用。

语境是由语言内部因素和语言外部因素共同组成的,或者分成上下文的小 语境和作为语言环境、文化背景和主观心理的大语境, 体现结构多样化, 层次 多重性的特点。在语言教学中, 人们常常把语言内部语境作为对象进行研究并 进行教学，但这远远是不够的。因为教学语境在时间、场合、对象、任务、心 理、文化等方面具有现场性和差别。另外，非现场交际语境或者虚拟语境也是 大量存在的。

语境是一个复杂的动态系统, 在二语教学过程中, 利用语境来完成教学任 务, 提高教学效率, 是必不可少的。但这种语境又是一种跨文化的语境, 其组 成因素并非是有序排列和运行的，而是具有多元、易变、开放等非线性特点。 汉语国际教育既是语言的转换, 也是思维方式和文化系统的转换。汉语国际教 育是跨文化交际活动, 这不仅要研究语言与文化的关系, 而且要从文化角度去 解释语言或从语言现象去解释文化; 不仅要比较语言间的异同, 而且要比较文 化间的异同, 还要将比较的结果结合起来解释文化或语言现象, 还要探讨文化 间或语言间的差异如何影响到语言学习和运用。

作为二语学习, 参与跨文化语境的两大基本对象是学习者和教师, 从汉语 国际教育来说,多元复杂的文化社会和学习者就是汉语教师所面对的跨文化语 境。目前, 部分外派到海外的教师或教师志愿者, 难以适应这种跨文化语境, 产生了文化障碍或文化冲突现象，给教学带来负面的影响; 还有的人在国内是 不错的汉语教师，但在海外却成绩平平，个别人甚至会提前终止自己的工作。 因此, 从浑沌学角度来审视, 会给汉语国际教育带来一定的启示。

宽容：既然汉语学习是一种非线性的过程，就不能简单地用有 “因” 必有 “果” 去解释, 那么, 它的不可预测性也就理所当然。教师应该对学习者体现 出一种宽容的态度。宽容学习者在学习过程中所犯的错误，从而给予更多的耐 心和帮助。理解学习者所受的挫折, 从而给予更多的鼓励和支持。教师在教学 过程中用移情的力量去感化学习者, 应该是优化教学情感氛围、增进师生互动 交流效果的极为良好的心理条件。

因材施教: 学生学习汉语的行为具有不可测性和差别性。比如在 “把字句” 这一语法学习过程中, 甲生可能起初糊涂, 后来慢慢理解了; 乙生则一开始表 现为比较清楚, 但接触了各种非规则变化后, 反而不清楚了。语言悟性高的学 生可能在一次顿悟中轻而易举掌握了规则, 而悟性差的可能事倍功半仍难以解 脱。教师在具体教学过程中应当善于发现和针对不同类型的学习者的初始敏感 性, 摸索并指导他们采取切合自身实际的、行之有效的学习策略。在教学方式、 
内容题材、作业辅导、练习份量和难易度、教学要求上, 采取差异化的措施, 努力达到最大限度的因材施教和教学个性化。

反思和探索: 教师要学会反思自己的价值观和文化观, 反思自己国家教育 体系和教育特点, 并要建立与之比较的关联性。比如说, 不能穿着旗袍在穆斯 林学校, 吃狗肉不能成为一种文化夸耀。严格的课堂纪律能否出现在欧美的课 堂上? 公布成绩有没有负面影响？跟家长告状适合在海外吗？浑沌学强调有 序和无序的辩证关系和整体性。第二语言习得是在熟悉和掌握母语前提下进行 的, 其语言学习背景与初学母语时差别很大, 原有语言习得机制（初始条件） 对第二语言产生怎样的敏感性作用，教师应当具有一种自觉的探索精神和意 在理解的态度。教师应着眼于发现和分析在许多表面的学习现象和学习者行为 背后潜藏着的因素、某种细微的差别变化、及学习过程中的其它“奇异吸引子”, 观察并求解发生在教学临场不可预测的事态及其千变万化的 “规则”, 以便充 分地作好教学准备, 化教学中的 “浑沌” 为教学中的 “有序”, 通过反馈控制 切实提高汉语教学效果与质量。

\section{5. 结语}

决定论视因果关系为常态, 浑沌学理论却认为 “浑沌” 才是自然和人文社 会的常态，任何事物和现象间常因交互而形成复杂的浑沌状态，即 “非线性”。 非线性可以理解为非必然、非逻辑、非确定、无序、不平衡, 但它没有完全排 斥因果关系, 只是更适合于可能尚未认识到的多因一果、一因多果、诸因诸果 的情景。汉语国际教育是复杂的跨文化语境教学, 既有一定的封闭性、规约性、 目标性和有序性，又具有开放性、不确定性、多变性和无序性，单纯依靠线性 思维或无视复杂型思维的存在, 不利于汉语国际教育的研究和进行。

\section{参考文献}

陈学军, 2004, 复杂性思维: 一种新的课堂教学组织观 $[\mathrm{J}]$ 。当代教育科学, (3) : 26-28。

丁梦阳, 2008 , 非线性科学对外语学习及教学的启示 $[\mathrm{J}]$ 。江苏教育学院学报, (1) : 99-112。

苗东升、刘华杰, 1994, 浑沌学纵横论 $[M]$ 。北京: 人民大学出版社。

莫海文, 2011, 二语习得: 一个复杂的非线性系统 $[J]$ 。山东外语教学，（3）: 91-95。 
王希杰, 深化对语言的认识, 促进语言科学的发展 $[\mathrm{J}]$ 。语言文字应用, 1994 (3) : 9-15。

吴平, 2005, 浑沌学和文化语言学: 方法论的思考 [A], 张公瑾、丁石庆主编, 浑沌学与语言文化研究 $[\mathrm{C}]$ 。北京: 中央民族大学出版社。

吴平, 2011, 第二语言习得中的浑沌/复杂性理论 [A], 浑沌学与语言文化研究 新动态一一国外相关研究译文集 $[\mathrm{C}]$ 。北京: 中央民族大学出版社。

吴平，2008，同素反序双音节名词中的浑沌现象分析 [A], 混沌学与语言文化 研究新视野 $[\mathrm{C}]$ 。北京: 中央民族大学出版社。

吴平，2012，对外汉语教学中的文化词语 [M]。北京：世界图书出版公司。 熊和平, 2005 , 复杂性思维与我国教学理论的创新 $[\mathrm{J}]$ 。课程 - 教材 - 教法, (2) : 20-26。

张公瑾, 1997, 走向 21 世纪的语言科学 $[J]$ 。民族语文，(2)：28-35。 张公瑾, 1997, 混沌学与语言研究 $[J]$ 。语言教学与研究, (3) : 61-65。 张公瑾、丁石庆, 2004, 文化语言学教程 [M]。北京：教育科学出版社。 张武江, 2004, 非线性动力学与语言研究 $[J]$ 。重庆工学院学报, (6): 146-149。

Ellis, R., 1999. The Study of Second Language Acquisition. 上海: 上海外语 教育出版社.

Freeman, L., 1997. Chaos/complexity science and Second Language Acquisition. Applied Linguistics 18, 2: 141-165.

,-- 1998 . On the scope of Second Language Acquisition research. Language Learning 48, 4: 551-56.

Lightbown, P.M., 1985. Great expectations: Second Language Acquisition research and classroom teaching. Applied Linguistics 6, 2: 173-189. 